Internat. J. Math. \& Math. Sci.

Vol. 24, No. 10 (2000) 667-672

S0161171200004853

(C) Hindawi Publishing Corp.

\title{
ON STRUCTURE OF CERTAIN PERIODIC RINGS AND NEAR-RINGS
}

\author{
MOHARRAM A. KHAN
}

(Received 22 November 1999 and in revised form 23 March 2000)

\begin{abstract}
The aim of this work is to study a decomposition theorem for rings satisfying either of the properties $x y=x^{p} f(x y x) x^{q}$ or $x y=x^{p} f(y x y) x^{q}$, where $p=p(x, y)$, $q=q(x, y)$ are nonnegative integers and $f(t) \in t \mathbb{Z}[t]$ vary with the pair of elements $x, y$, and further investigate the commutativity of such rings. Other related results are obtained for near-rings.
\end{abstract}

Keywords and phrases. Distributively generated near-ring, $J$-ring, nil ring, periodic nearring, zero-commutativity.

2000 Mathematics Subject Classification. Primary 16Y30, 16U80.

1. Introduction. Searcóid and MacHale [10] established the commutativity of rings in which all products of two elements are potent. Recently, using this result Ligh and Luh [9] proved that such rings are direct sum of $J$-rings (i.e., rings satisfying Jacobson's $x^{n(x)}=x$ property (see [8])) and zero rings. More recently, Bell and Ligh [5] studied the direct sum decomposition of rings satisfying the property $x y=(x y)^{2} f(x, y)$, where $f(X, Y) \in \mathbb{Z}\langle X, Y\rangle$, the ring of polynomials in two noncommuting indeterminates. Now, we consider the following ring properties.

(P) For each $x, y$ in a ring $R$, there exist integers $p=p(x, y) \geq 0, q=q(x, y) \geq 0$ and a polynomial $f(t) \in t \mathbb{Z}[t]$ such that

$$
x y=x^{p} f(x y x) x^{q} .
$$

$\left(\mathrm{P}_{1}\right)$ For each $x, y$ in a ring $R$, there exist integers $p=p(x, y) \geq 0, q=q(x, y) \geq 0$ and a polynomial $f(t) \in t \mathbb{Z}[t]$ such that

$$
x y=x^{p} f(y x y) x^{q} .
$$

2. A decomposition theorem for rings. In this section, we establish a decomposition theorem which in turn allows us to study the commutativity of such rings. Throughout this section, $R$ represents an associative ring (may be without unity 1 ), and $C=N(R)$, the set of nilpotent elements of $R$. A ring $R$ is called periodic if for each $x \in R$, there exist distinct positive integers $m=m(x), n=n(x)$ such that $x^{m}=x^{n}$. A ring $R$ is called zero commutative if $x y=0$ implies that $y x=0$ for all $x, y \in R$. An element $x$ of $R$ satisfying the property $x^{n(x)}=x$ for some $n(x)>1$ is called potent. Let $B$ be the set of all potent elements. If $B=R$, then $R$ is a $J$-ring. By a well-known theorem of Jacobson [8], $J$-rings are necessarily commutative. A sufficient condition for $R$ to be periodic is Chacron's criterion: for each $x \in R$ there exists an integer 
$p=p(x)>1$ and a polynomial $f(t) \in \mathbb{Z}[t]$ such that $x^{p}=x^{p+1} f(x)$ (see [6]). Also it is shown in [2] that if $R$ is periodic, then every element $x \in R$ can be written in the form $x=b+c$, where $b \in B$ and $c \in C$. Further, Bell [4] remarked that if, in a periodic ring $R$, each element has a unique representation as above, then both $B$ and $C$ are ideals and $R=B \oplus C$. Motivated by these, we obtain a decomposition theorem for rings satisfying one of the properties $(\mathrm{P})$ and $\left(\mathrm{P}_{1}\right)$. In fact, we establish the following result.

THEOREM 2.1. Let $R$ be a ring satisfying one of the properties $(P)$ and $\left(P_{1}\right)$. Then $R$ is a direct sum of a J-ring and a nil ring.

Proof OF TheOrem 2.1. We break the proof into the following parts called steps.

STEP 1. Let $R$ be a ring satisfying (P). Then $R$ is periodic.

Proof. Take $y=x$ in (P). This shows that $R$ satisfies Chacron's criterion for periodicity and hence the ring satisfying $(\mathrm{P})$ is necessarily periodic.

STEP 2. Let $R$ be a ring satisfying (P). Then $R$ is zero-commutative.

Proof. Let $x y=0$. Then there exist integers $p^{\prime}=p(y, x) \geq 0, q^{\prime}=q(y, x) \geq 0$ and a polynomial $h(t) \in t \mathbb{Z}[t]$ such that $y x=y^{p^{\prime}} h(y x y) y^{q^{\prime}}=0$. This implies that $R$ is a zero-commutative.

STEP 3. Let $R$ be a ring satisfying (P). Then $R C=C R=\{0\}$.

Proof. Let $r(x)=2$. Replacing $y$ by $x$ in (P), we get $x^{2}=x^{r} g(x)$, for some $g(t) \in$ $t \mathbb{Z}[t]$ and, by Step $1, R$ is periodic; clearly $R$ is nil.

Next we have

$$
x^{2}=x^{r} g(x) \text { for } g(t) \in \mathbb{Z}[t], \quad r(x)>2 .
$$

Let $c \in C$ and $x \in R$. Then choose integers $p_{1}=p(c, x) \geq 0, q_{1}=q(c, x) \geq 0$ and a polynomial $f_{1}(t) \in t \mathbb{Z}[t]$ such that

$$
c x=c^{p_{1}} f_{1}(c \times c) c^{q_{1}} .
$$

From the equality (2.1), one can easily observe that $c^{2}=0$, and hence $0=x c^{2}=(x c) c$. Step 2 gives that $c(x c)=0$, which together with (2.2), yields that $c x=0$; and again Step 2 gives that $x c=0$ for all $x \in R, c \in C$. This gives the required result, that is,

$$
R C=C R=\{0\} .
$$

By Step $1, R$ is periodic so that each element $x \in R$ can be written in the form $b+c$, where $b \in B$, and $c \in C$. By a nice result of Bell [4], it is enough to show that this representation is unique. If $a+c=b+d$ for some $a, b \in B$ and $c, d \in C$, then

$$
a-b=d-c .
$$

Let $a, b \in B$. Then there exist at least one odd of the positive integers $r=r(a)$ and $s=s(b)$ such that $a^{r}=a$ and $b^{s}=b$. 
Let $k=(r-1) s-(r-2)=(s-1) r-(s-2)$ be an odd positive integer. Thus it is clear that $a^{k}=a$ and $b^{k}=b$. Also $e_{1}=a^{k-1}, e_{2}=b^{k-1}$ are idempotents in $R$ with $e_{1} a=a$ and $e_{2} b=b$. Multiplying (2.4) by $a$ and $b$ from both sides and using the result of Step 3, we get $a^{2}=a b=b a$ and $b^{2}=a b=b a$. This gives that $a^{2}=b^{2}$ and hence $e_{1}=e_{2}$.

If $k$ is even and $a^{k}=a$, then $a^{2(k-1)+1}=a$, where $2(k-1)+1$ is odd, so this yields the required result.

Left-multiplying (2.4) by $e_{1}$ now yields $a=b$, and this completes the proof.

Similar arguments can be used if $R$ satisfies the property $\left(\mathrm{P}_{1}\right)$.

REMARK 2.2. By a careful scrutiny of the result of Step 2, one concludes that the nilpotent elements of $R$ annihilate $R$ on both sides and hence are central. However, $J$-rings are commutative, so that Theorem 2.1 at once gives the following corollary which extends the main results of $[10,12]$.

COROLlaRY 2.3. Let $R$ be a ring satisfying any one of the properties $(P)$ and $\left(P_{1}\right)$. Then $R$ is commutative.

3. Decomposition theorems for near-rings. In this section, we investigate the structure of near-rings satisfying properties $(\mathrm{P})$ and $\left(\mathrm{P}_{1}\right)$. Here, $R$ denotes a left near-ring and $Z=Z(R)$ the multiplicative center of $R$. An element $x$ of $R$ is called distributive if $(a+b) x=a x+b x$ for all $a, b \in R$. If all the elements of $R$ are distributive, then $R$ is called a distributive near-ring. A near-ring $R$ is called a periodic near-ring if for each $x \in R$, there exist distinct positive integers $m=m(x), n=n(x)$ such that $x^{m}=x^{n}$. A near-ring $R$ is called a zero-symmetric if $0 x=0$ for all $x \in R$ (left distributivity yields $x 0=0$ ).

An ideal of a near-ring $R$ is a normal subgroup $I$ of $(R,+)$ such that (i) $R I \subseteq I$ and (ii) $(x+\alpha) y-x y \in I$ for all $x, y \in R$ and $\alpha \in I$ (see [11] for details).

It is natural to ask the question: do the analogous hypotheses give the direct sum decomposition in the case of near-rings?

Example 29, number (2.5) due to Clay [7] guarantees that one cannot get the direct sum decomposition under the hypotheses of the above theorem, even in the case of distributive near-rings.

Following [5], we define a weaker notion of orthogonal sum: a near-ring $R$ is an orthogonal sum of sub-near-rings $P$ and $Q$, denoted by $R=P+Q$, if $P Q=Q P=\{0\}$ and each element of $R$ has a unique representation of the form $p+q, p \in P, q \in Q$.

Now, our aim is to establish the decomposition theorems for near-rings satisfying any one of the following related properties:

$\left(\mathrm{P}_{2}\right)$ For each pair of elements $x, y \in R$, there exist integers $p=p(x, y) \geq 0, q=$ $q(x, y) \geq 0$, and $r=r(x, y) \geq 1$ such that

$$
x y=x^{p}(x y x)^{r} x^{q} .
$$

$\left(\mathrm{P}_{3}\right)$ For each pair of elements $x, y \in R$, there exist integers $p=p(x, y) \geq 0, q=$ $q(x, y) \geq 0$, and $r=r(x, y)>1$ such that

$$
x y=x^{p}(y x y)^{r} x^{q} .
$$


THEOREM 3.1. Let $R$ be a near-ring satisfying the property $\left(P_{2}\right)$. If the idempotents of $R$ are multiplicatively central, then $B$ is a sub-near-ring with $(B,+)$ abelian and $C$ is a sub-near-ring with trivial multiplication and $R=C \dot{+} B$.

Before proving our theorem, we state the following known results.

LEMMA 3.2 (see [1]). Let $R$ be a zero-commutative near-ring. Then the set $C$ of nilpotent elements is an ideal if and only if $C$ is a subgroup of the additive group $(R,+)$.

LEMMA 3.3 (see [3]). Let $R$ be a periodic near-ring with multiplicative identity. If $C \subseteq$ $Z$, then $(R,+)$ is abelian.

LEMMA 3.4 (see [5]). Let $R$ be a near-ring in which the idempotents are multiplicatively central. If $e_{1}$ and $e_{2}$ are idempotents, then there exists an idempotent $e_{3}$ such that $e_{3} e_{1}=e_{1}$ and $e_{3} e_{2}=e_{2}$.

Now, we prove the following.

LEMMA 3.5. Let $R$ be a near-ring satisfying $\left(P_{2}\right)$. Then the set $C$ of nilpotent elements of $R$ is an ideal.

Proof. Obviously, we see that a near-ring satisfying $\left(\mathrm{P}_{2}\right)$ is necessarily zero-symmetric as well as zero-commutative. Let $c \in C$ and $x$ an arbitrary element of $R$. Then there exist integers $p=p(x, c) \geq 0, q=q(x, c) \geq 0$, and $r=r(x, c)>1$ such that

$$
x c=x^{p}(x c x)^{r} x^{q} .
$$

Next, choose integers $p^{\prime}=p(x) \geq 0, q^{\prime}=q(x) \geq 0$, and $r^{\prime}=r(x)>1$ such that

$$
x^{2}=x^{p^{\prime}+q^{\prime}+3 r^{\prime}} \text {. }
$$

Since (3.4) gives that $c^{2}=0$ for any $c \in C$, we obtain that $c(c x)=c^{2} x=0$ and the zero-commutativity in $R$ yields that $(c x) c=0$. Thus, by using (3.3), we find that $x c=0$ for all $x \in R$, and also zero-commutativity of $R$ implies that $c x=0$, that is,

$$
R C=C R=\{0\} .
$$

Equation (3.5) shows that the nilpotent elements of $R$ annihilate $R$ on both sides and hence, in particular, $C^{2}=\{0\}$ and $C \subseteq Z$. If $c, d \in C$, then $(c-d)^{2}=0$. This gives that $c-d \in C$ and $C$ is a sub-group of the additive group $(R,+)$. Now the application of Lemma 3.2 yields the required result.

LEMMA 3.6. Let $R$ be a near-ring satisfying the property $\left(P_{2}\right)$. If the idempotents of $R$ are multiplicatively central, then $B$ is a sub-near-ring with $(B,+)$ abelian.

Proof. Let $a, b \in B$. Then there exist integers $m^{\prime}=m(a)>1$ and $n^{\prime}=n(b)>1$ such that $a^{m^{\prime}}=a$ and $b^{n^{\prime}}=b$. If

$$
s=\left(m^{\prime}-1\right) n^{\prime}-\left(m^{\prime}-2\right)=\left(n^{\prime}-1\right) m^{\prime}-\left(n^{\prime}-2\right)>1,
$$

then it is clear that $a^{s}=a$ and $b^{s}=b$. Note also that $e_{1}=a^{s-1}$ and $e_{2}=b^{s-1}$ are central idempotents in $R$ with $e_{1} a=a$ and $e_{2} b=b$. Also, in view of $\left(\mathrm{P}_{2}\right)$, we find that

$$
a b=\left(e_{1} a\right)\left(e_{2} b\right)=\left(e_{1} e_{2}\right)(a b)=\left(e_{1} e_{2}\right)^{p}\left(e_{1} e_{2} a b e_{1} e_{2}\right)^{r}\left(e_{1} e_{2}\right)^{q}
$$


for some integers $p=p\left(e_{1} e_{2}, a b\right) \geq 0, q=q\left(e_{1} e_{2}, a b\right) \geq 0$, and $r=r\left(e_{1} e_{2}, a b\right)>1$. This yields that

$$
a b=e_{1} e_{2}(a b)^{r} e_{1} e_{2}
$$

so, $a b \in B$. Moreover, since $R / C$ has the $x^{n}=x$ property, we have an integer $k>1$ such that

$$
(a-b)^{k}=a-b+c
$$

where $a, b \in B$ and $c \in C$. Now $e_{1}$ and $e_{2}$ are central idempotents in $R$ and, in view of Lemma 3.4, there exists an idempotent $e \in R$ such that $e e_{1}=e_{1}$ and $e e_{2}=e_{2}$. This implies that $e a=a$ and $e b=b$. Since (3.5) is still valid in the present situation, multiply (3.9) by $e$ to get $(a-b)^{k}=a-b$, and hence $a-b \in B$. Also, $e R$ is a periodic near-ring with multiplicative identity element in which nilpotent elements are multiplicatively central. Thus by Lemma 3.3, $(e R,+)$ is abelian. Therefore, $e a+e b=e b+e a$, that is, $a+b=b+a$, and hence $(B,+)$ is abelian.

Proof OF TheOrem 3.1. Let $x \in R$. Then in view of (3.4), if $x^{2}=x^{k}, k=p^{\prime}+q^{\prime}+$ $3 r^{\prime} \geq 3$, then clearly $x^{j}=x^{j+s(k-2)}$ for all $j \geq 2$ and $s \geq 1$ it follows at once that $\left(x^{k-1}\right)^{k-1}=x^{k-1}$; so $x^{k-1} \in B$; it also follows that $\left(x-x^{k-1}\right)^{2}=0$ and $x-x^{k-1} \in C$. Hence, we can write $x=x-x^{k-1}+x^{k-1}$ and see that $R=C+B$. Now, in view of Lemmas 3.5 and 3.6, it remains only to show that each element of $R$ has the unique representation in the form $c+b$, where $c \in C, b \in B$. Suppose that $c+a=d+b$, where $c, d \in C$ and $a, b \in B$. Then $-d+c=b-a \in C \cap B=\{0\}$. This gives that $a=b$ and $c=d$.

REMARK 3.7. In view of Example E-14 [11, page 340], it is clear that the centrality of idempotents in the hypotheses of Theorem 3.1 is not superfluous.

REMARK 3.8. If a near-ring $R$ satisfies $\left(\mathrm{P}_{3}\right)$, then it can be easily verified that $R$ need not be zero-commutative. However, a zero-symmetric near-ring satisfying $\left(\mathrm{P}_{3}\right)$ is necessarily zero-commutative. Hence, for a zero-symmetric near-ring satisfying $\left(\mathrm{P}_{3}\right)$, Lemmas 3.5 and 3.6 may be proved easily in the same fashion. By using similar arguments used to prove Theorem 3.1, with necessary variations, we can prove the following result. We omit the details of the proof to avoid repetition.

THEOREM 3.9. Let $R$ be a zero-symmetric near-ring satisfying $\left(P_{3}\right)$. If the idempotent elements of $R$ are multiplicatively central, then $C$ is a sub-near-ring with trivial multiplication, $B$ is a sub-near-ring with $(B,+)$ abelian and $R=C \dot{+} B$.

Acknowledgement. The author is indebted to the learned referee for his generous help and valuable suggestions.

\section{REFERENCES}

[1] H. E. Bell, Certain near-rings are rings, J. London Math. Soc. (2) 4 (1971), 264-270. MR 45\#1979. Zbl 223.16020.

[2] _ A commutativity study for periodic rings, Pacific J. Math. 70 (1977), no. 1, 29-36. MR 58\#793. Zbl 364.16012. 
[3] C Centres for near-rings: applications to commutativity theorems, Proc. Edinburgh Math. Soc. (2) 23 (1980), no. 1, 61-67. MR 82a:16034. Zbl 433.16026.

[4] _ On commutativity and structure of periodic rings, Math. J. Okayama Univ. 27 (1985), 1-3. MR 87g:16049. Zbl 604.16026.

[5] H. E. Bell and S. Ligh, Some decomposition theorems for periodic rings and near-rings, Math. J. Okayama Univ. 31 (1989), 93-99. MR 91i:16053. Zbl 696.16029.

[6] M. Chacron, On a theorem of Herstein, Canad. J. Math. 21 (1969), 1348-1353. MR $41 \# 6905$. Zbl 213.04302.

[7] J. R. Clay, The near-rings on groups of low order, Math. Z. 104 (1968), 364-371. MR 37\#258. Zbl 153.35704.

[8] N. Jacobson, Structure theory for algebraic algebras of bounded degree, Ann. of Math. (2) 46 (1945), 695-707. MR 7,238c. Zbl 060.07501.

[9] S. Ligh and J. Luh, Direct sum of J-rings and zero rings, Amer. Math. Monthly 96 (1989), no. 1, 40-41. MR 90a:16021. Zbl 671.16015.

[10] M. Ó Searcóid and D. MacHale, Two elementary generalisations of Boolean rings, Amer. Math. Monthly 93 (1986), no. 2, 121-122. MR 87g:16055. Zbl 601.16025.

[11] G. Pilz, Near-Rings, The theory and its applications, 2nd ed., North-Holland Mathematics Studies, no. 23, pp. xv+470, North-Holland Publishing Co., Amsterdam, New York, Oxford, 1983. MR 85h:16046. Zbl 521.16028.

[12] H. Tominaga and A. Yaqub, Commutativity theorems for rings with constraints involving a commutative subset, Resultate Math. 11 (1987), no. 1-2, 186-192. MR 88k:16031. Zbl 618.16029.

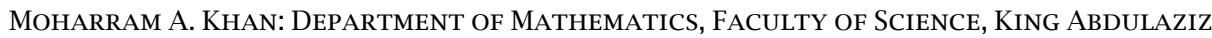
UNIVERSITY, P.O. BOX 30356, JEDDAH-21477, SAUDI ARABIA

E-mail address: nassb@hotmai1. com 


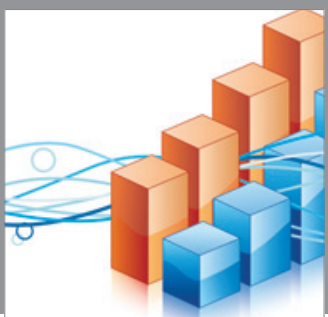

Advances in

Operations Research

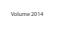

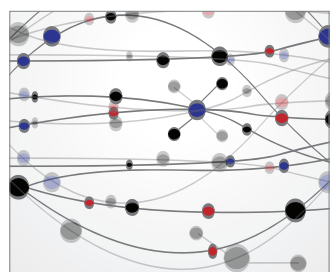

\section{The Scientific} World Journal
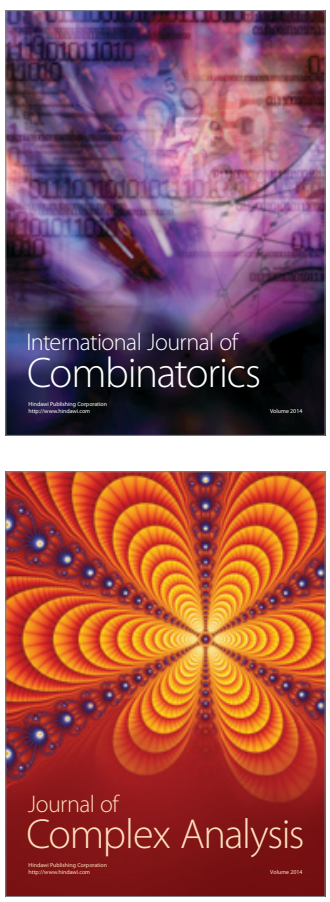

International Journal of

Mathematics and

Mathematical

Sciences
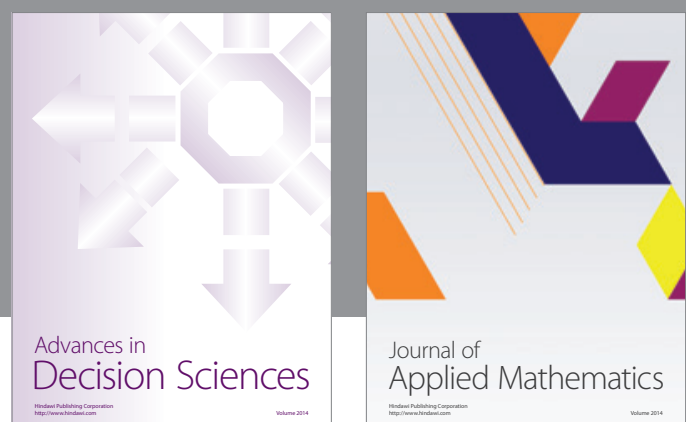

Journal of

Applied Mathematics
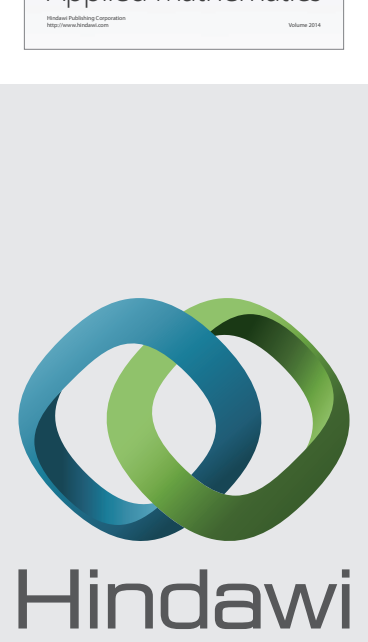

Submit your manuscripts at http://www.hindawi.com
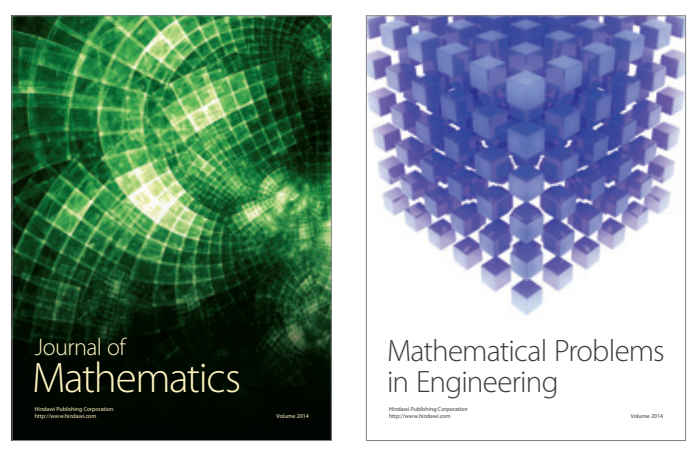

Mathematical Problems in Engineering
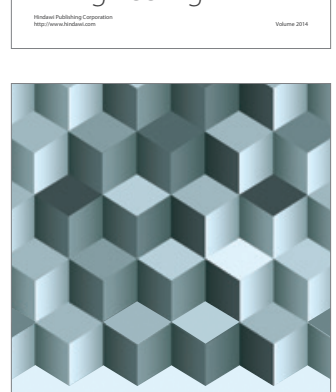

Journal of

Function Spaces
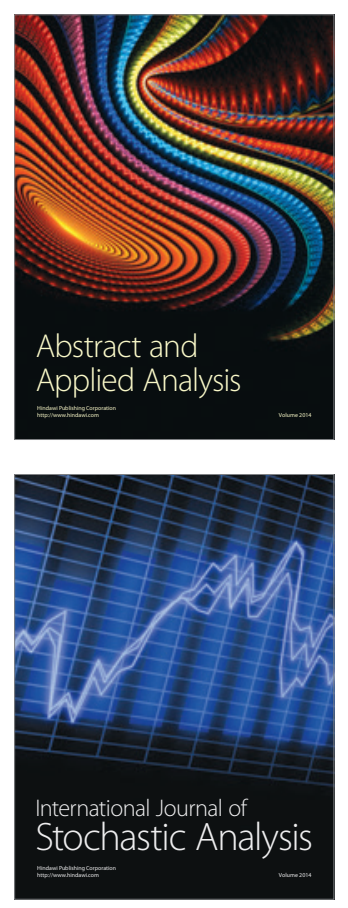

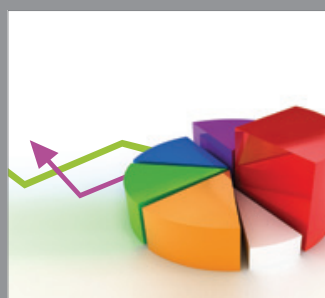

ournal of

Probability and Statistics

Promensencen
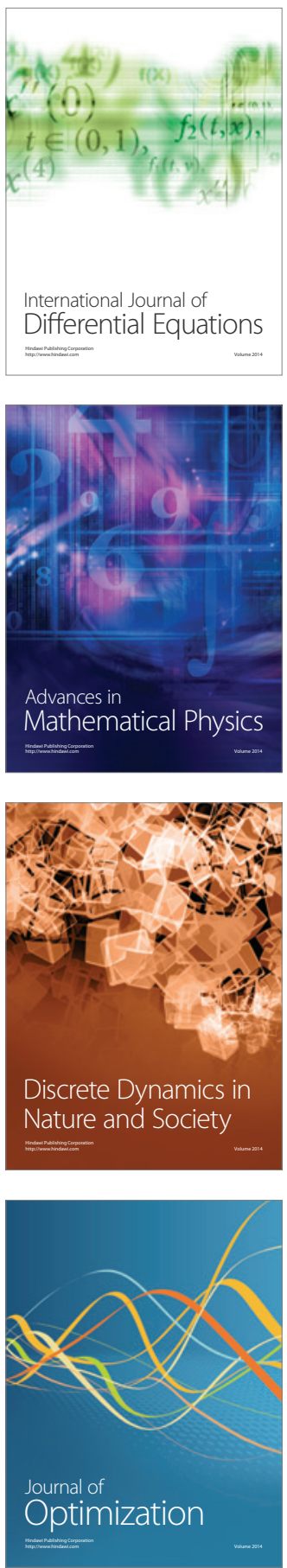